\title{
1,3-BIS(2-HYDROXYIMINOMETHYLPYRIDINIUM) PROPANE AS THE POTENTIAL REACTIVATOR OF THE ACETYLCHOLINESTERASE INHIBITED BY NERVE AGENTS
}

\author{
Kamil Kuča ${ }^{1}$, Vlastimil Dohnal ${ }^{2}$ \\ Purkyně Medical Military Academy in Hradec Králové, Czech Republic: Department of Toxicology ${ }^{1}$; Masaryk University \\ in Brno, Faculty of Science, Czech Republic: Department of Analytic Chemistry² \\ Summary: The oxime K005 [1,3-bis(2-hydroxyiminomethylpyridinium) propane dibromide] for the reactivation of the en- \\ zyme acetylcholinesterase (AChE) inhibited by cyclosarin and VX was tested. Its reactivation potency was not better in \\ comparison with the currently used $\mathrm{AChE}$ reactivator - pralidoxime. On the other hand, the oxime K005 has its maximum \\ reactivation ability at the concentration $10^{-4} \mathrm{M}$, which could be achieved for human use. The maximum concentration of \\ pralidoxime was reached at the concentrations $10^{-1} \mathrm{M}$ (for cyclosarin) and $10^{-3} \mathrm{M}$ (for VX) but these concentrations are \\ not available for the use in vivo.
}

Key words: Acetylcholinesterase; Reactivation; Inhibition; Oximes; Reactivator; Pralidoxime; Cyclosarin; VX; In vitro; K005; Nerve agents; Organophosphates

\section{Introduction}

Acetylcholinesterase (AChE; EC 3.1.1.7) reactivators and parasympatolytics are currently used as the antidotes for the treatment of the organophosphate compounds (OPCs) intoxications (10). OPCs are largely used in agriculture (insecticides), in industry and technology (softening agents) and in military technology (chemical warfare agents) (4). OPCs affect AChE as irreversible inhibitors. They phosphorylate or phosphonylate the serine hydroxyl group at the active site of the enzyme (6). Due to the inhibition, AChE cannot be physiologically functional (10).

Pralidoxime [1-methyl-2-hydroxyiminomethylpyridinium chloride], obidoxime [1,3-bis(4-hydroxyiminomethylpyridinium)-2-oxa-propane dichloride] and H-oxime HI-6 [1-(2-hydroxyiminomethylpyridinium)-3-(4-carbamoylpyridinium)-2-oxa-propane dichloride] are currently considered to be the main representatives of the AChE reactivators (5). These compounds have in their molecules incorporated characteristic features: the presence of the quaternary nitrogen (in the case of obidoxime and HI-6 even two quaternary nitrogen atoms), functional aldoxime group and in the case of bisquaternary pyridinium aldoximes, the connecting chain of the specific length and shape (9).

None from the above mentioned $\mathrm{AChE}$ reactivators are able to satisfactorily reactivate $\mathrm{AChE}$ inhibited by all kinds of OPCs, especially by nerve agents such as tabun, sarin or cyclosarin (5).

The reactivation insufficiency is caused by the differences in the structure of the AChE inhibitors (3). According to the insufficient reactivation potency of all currently used reactivators, scientists in many laboratories throughout the world synthesize and test new AChE reactivators, with the aim to treat intoxications by all known OPCs (11).

In our Department we have synthesized 1,3-bis(2-hydroxyiminomethylpyridinium) propane dibromide (called K005) as the potential reactivator of the AChE inhibited by nerve agents (7). In this work we have tested the potency to reactivate AChE inhibited by highly toxic nerve agents such as cyclosarin and VX.

\section{Material and Methods}

\section{Chemical}

Compound K005 [1,3-bis(2-hydroxyiminomethylpyridinium) propane dibromide] was prepared earlier at our department (7) by the modification of the synthesis described by Berry et al. (1). Pralidoxime [1-methyl-2-hydroxyiminomethylpyridinium chloride] was used for comparison as the currently used AChE reactivator. Purities of the tested AChE reactivators were detected using HPLC. Nerve agents (cyclosarin and VX) were obtained from the Military Facility Brno (97\% purity). All other chemicals used were of reagent grade (Aldrich). 


\section{Enzyme}

Rat brain acetylcholinesterase was chosen as the source of the enzyme. Its preparation was as follows. Lightly ethernarcotized animals were killed by bleeding from a carotid artery and the brains were removed, washed with saline and homogenized in an Ultra-Turrax homogenizer in a distilled water to make a $10 \%$ homogenate.

Tab. 1: Kinetic parameters for the reactivation of cyclosarin inhibited AChE.

\begin{tabular}{|l|c|c|c|}
\hline Oxime & $\mathrm{K}_{\mathrm{R}}[\mu \mathrm{M}]$ & $\mathrm{k}_{\mathrm{R}}\left[\mathrm{min}^{-1}\right]$ & $\mathrm{k}_{\mathrm{r}}\left[\mathrm{min}^{-1} \mathrm{M}^{-1}\right]$ \\
\hline 2-PAM & 12000 & 0.040 & 3 \\
\hline K005 & 5 & 0.010 & 2196 \\
\hline
\end{tabular}

Tab. 2: Kinetics parameters for the reactivation of VX inhibited AChE.

\begin{tabular}{|l|c|c|c|}
\hline Oxime & $\mathrm{K}_{\mathrm{R}}[\mu \mathrm{M}]$ & $\mathrm{k}_{\mathrm{R}}\left[\mathrm{min}^{-1}\right]$ & $\mathrm{k}_{\mathrm{r}}\left[\mathrm{min}^{-1} \mathrm{M}^{-1}\right]$ \\
\hline 2-PAM & 127 & 0.047 & 370 \\
\hline K005 & 1350 & 0.132 & 98 \\
\hline
\end{tabular}

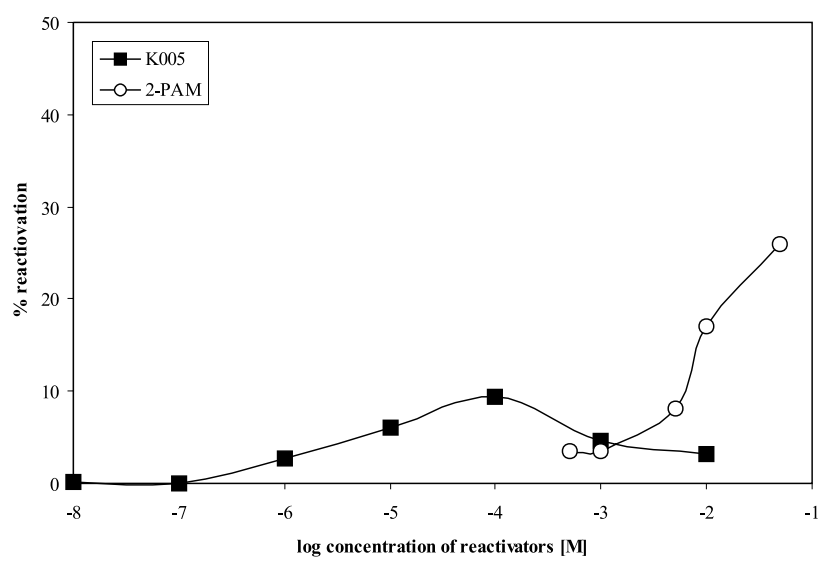

Fig. 1: Concentration-reactivation relationship for the reactivation of cyclosarin inhibited AChE.

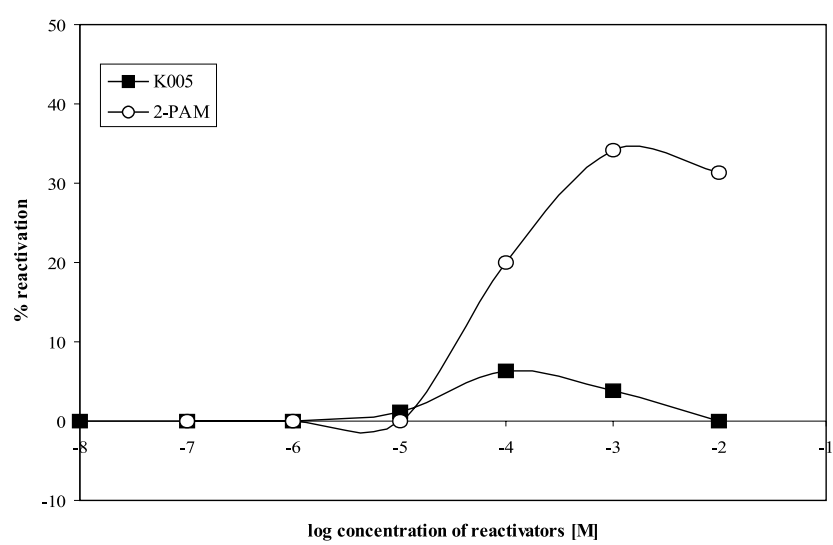

Fig. 2: Concentration-reactivation relationship for the reactivation of VX inhibited AChE.

\section{In vitro measurement}

Reactivation effectivities of the oximes were tested in vitro on the model of AChE inhibited by cyclosarin using standard reactivation test with electrometric instrumentation (8). The AChE homogenate $(0.5 \mathrm{ml})$ was mixed with $0.5 \mathrm{ml}$ of $0.01 \mu \mathrm{M}$ cyclosarin in dry isopropanol and incubated for $30 \mathrm{~min}\left(25^{\circ} \mathrm{C}\right)$. Then $2.5 \mathrm{ml}$ of $3 \mathrm{M} \mathrm{NaCl}$ was added and supplied by distilled water to a volume of $23 \mathrm{ml}$. After that, $2 \mathrm{ml}$ of $0.02 \mathrm{M}$ acetylcholine bromide was added and enzyme activity was assayed titrimetrically at $\mathrm{pH} 8.0$ and $25^{\circ} \mathrm{C}$ on the Autotitrator RTS 822 (Radiometer, Denmark).

The activities of intact $\left(a_{o}\right)$ and GF-inhibited $\left(a_{i}\right)$ AChE were determined. When GF-inhibited AChE was incubated 10 min with solution of reactivator, the activity of reactivated AChE $\left(a_{r}\right)$ was obtained. The activity values $\mathrm{a}_{0}, \mathrm{a}_{\mathrm{i}}$ and $\mathrm{a}_{\mathrm{r}}$ were calculated from the slopes of the initial part of titration curves. Each value represents arithmetic mean from two independent measurements.

\section{Results}

\section{Affinity to the intact enzyme}

Constant $K_{D I S}$ characterizes the affinity of the AChE reactivators to the intact enzyme. The lower is its value, the higher is its affinity to the intact enzyme. In our case, compound K005 has value of the dissociation constant four times lower $(53 \mu \mathrm{M})$ compared to pralidoxime $(210 \mu \mathrm{M})$. Thus, its affinity to the intact enzyme is higher.

\section{Reactivation of cyclosarin inhibited AChE}

The results obtained for the cyclosarin inhibited AChE are summarized in Table 1 . Values of the constant $k_{R}$ (characterizing the decomposition of the inhibited enzyme-reactivator complex) and $k_{r}$ (characterizing velocity of overall reaction) favour compound $\mathrm{K} 005$. On the other hand, $K_{R}$ (characterizing the affinity of the enzyme to the inhibited $\mathrm{AChE}$ ) is advantageous for the pralidoxime. The relationship between the concentration of the oxime (concentration range from $10^{-8}$ to $10^{-1} \mathrm{M}$ ) and reactivation potency of the oximes is shown in Figure 1. The concentration with the maximum reactivation potency for the oxime K005 is at the concentration $10^{-4} \mathrm{M}(10 \%)$. On the other hand, pralidoxime is able to reactivate cyclosarin-inhibited AChE in vitro in very high concentrations, and therefore, it would be toxic for the use in vivo.

\section{Reactivation of VX inhibited AChE}

Kinetics parameters characterizing the ability of the oxime K005 and pralidoxime to reactivate VX-inhibited $\mathrm{AChE}$ are summarized in Table 2. Both reactivators were able to reactivate VX-inhibited AChE. Value of the constant $K_{R}$ characterizing affinity to the inhibited enzyme favours pralidoxime. Its afinity to the inhibited enzyme is almost 11 times higher compared to the oxime K005. Constant $k_{R}$ characterizing the decomposition of the inhibited enzymereactivator complex is about three times higher for the oxi- 
me K005. On the other hand, constant $k_{r}$ characterizing the velocity of the overall reaction favours pralidoxime and its value is about 3.5 times higher compared to oxime K005.

The concentration-reactivation relationship is demonstrated in the Figure 2. $10^{-3} \mathrm{M}$ concentration of the pralidoxime is necessary to reach $34 \%$ reactivation of $\mathrm{VX}$ inhibited AChE. In the case of the oxime K005, maximum reactivation potency $(6.35 \%)$ is reached at the concentration $10^{-4} \mathrm{M}$.

\section{Discussion}

Oxime K005 does not reach better reactivation potency than pralidoxime in the case of cyclosarin and VX-inhibited AChE. On the other hand, concentration with maximum reactivation potency was reached at the concentration lower than that in the case of the pralidoxime. These concentrations could be safe for human use (8).

Reactivation of inhibited AChE depends on the inhibitors used and on the chemical structure of the reactivators $(3,5,8)$. Our results confirm these observations. There are differences in the course of the reactivation curves of the oxime K005 or pralidoxime for inhibition by cyclosarin or VX.

Shape of the molecules of the AChE reactivators affects reactivation process, too $(2,9)$. According to our results, there were significant differences in the reactivation potency between oxime K005 and pralidoxime. Pralidoxime has in its molecule one quaternary nitrogen only while oxime K005 has two quaternary niotrogens. The presence of the quaternary nitrogen in the molecule is an important factor for reactivation process (12).

In conclusion, we have tested oxime K005 [1,3-bis(2hydroxyiminomethylpyridinium) propane dibromide] for the reactivation of the enzyme AChE inhibited by cyclosarin and VX. Its reactivation potency was not better in comparison with currently used AChE reactivator - pralidoxime. On the other hand, oxime K005 has maximum reactivation ability at the concentration $10^{-4} \mathrm{M}$, which could be safe for human use. Maximum concentration of pralidoxime was reached at the concentrations $10^{-1} \mathrm{M}$ (for cyclosarin) and $10^{-3} \mathrm{M}$ (for VX). Nevertheless, these concentrations are not available for the use in vivo.

\section{Acknowledgement}

The authors are grateful to Mrs. I. Ježková and Mrs. M. Hrabinová for their skilful technical assistance. The study was supported by the grant of Ministry of Defense No. ONVLAJEP20031.

\section{References}

1. Berry WK, Davies DR, Green AL. Oximes of $\alpha, \omega$-diquaternary alkane salts as antidotes to organophosphate anticholinesterases. Brit J Pharmacol 1959;14: 186-91.

2. Bielavský J, Kassa J, Elsnerová E, Dejmek L. Cholinesterase reactivators derived from pyridine-2-carbaldoxime. Collect Czech Chem Commun 1997;63:199-204.

3. Cabal J, Kassa J, Patočka J. Inhibition of plasma cholinesterase by O-alkylfluorophosphonates. Collect Czech Chem Commun 1996;62:521-6.

4. Kassa J. Review of oximes in the antidotal treatment of poisoning by organophosphorus nerve agents. J Toxicol Clin Toxicol 2002;40:803-16.

5. Kuča K, Bielavský J, Cabal J, Bielavska M. Synthesis of a potential reactivator of acetylcholinesterase - 1-(4-hydroxyiminomethylpyridinium)-3-(carbamoylpyridinium)-propane dibromide. Tetrahedron Lett 2003;44:3123-5.

6. Kuča K, Bielavský J, Cabal J, Kassa J. Synthesis of a New Reactivator of tabuninhibited acetylcholinesterase. Bioorg Med Chem Lett 2003;13:3545-7.

7. Kuča K, Cabal J, Patočka J, Kassa J. Synthesis of bisquaternary symmetric - $\chi, \delta$ bis(2-hydroxyiminomethylpyridinium)alkane dibromides and their reactivation of cyclosarin-inhibited acetylcholinesterase. Lett Org Chem 2004;1:84-6.

8. Kuča K, Kassa J. A comparison of the ability of a new byispyridinium oxime 1-(4-hydroxyiminomethylpyridinium)-4-(4-carbamoylpyridinium)butane dibromide and currently used oximes to reactivate nerve agent-inhibited rat brain acetylcholinesterase by in vitro methods. J Enzyme Inhib Med Chem 2003:18:529-35.

9. Kuča K, Patočka J, Cabal J. Reactivation of organophosphate inhibited acetylcholinesterase activity by $\alpha, \omega$-bis-(4-hydroxyiminomethylpyridinium)alkanes in vitro. J Appl Biomed 2003;1:207-11.

10. Marrs TC. Organophosphate poisoning. Pharmacol Therap 1993;58:51-66.

11. Petrova I, Bielavský J. An overview of syntheses of cholinesterase reactivators from 1980 to 1992. Voj Zdrav Listy 2001;70:63-73 (in Czech).

12. Ševčíková K, Hampl F, Liška F, Cabal J, Patočka J. Sustituted pyridinium derivatives as inhibitors of acetylcholinesterase. Voj Zdrav Listy - Suplementum 1998;67:28-31.

Submitted October 2003.

Accepted June 2004.

Ing. Kamil Kuča,

Purkyně Medical Military Academy,

Department of Toxicology,

Třebešská 1575, 50001 Hradec Králové,

Czech Republic.

e-mail: kucakam@pmfhk.cz 